\title{
X-ray dynamical diffraction from partly relaxed epitaxial structures
}

\author{
A. I. Benediktovich, ${ }^{1}$ I. D. Feranchuk, ${ }^{1}$ and A. Ulyanenkov ${ }^{2}$ \\ ${ }^{1}$ Department of Theoretical Physics, Belarusian State University, 4 Nezavisimosti Av., 220030 Minsk, Republic of Belarus \\ ${ }^{2}$ Bruker AXS GmbH, Östliche Rheinbrückenstr. 49, 76187 Karlsruhe, Germany
}

(Received 26 May 2009; revised manuscript received 8 September 2009; published 15 December 2009)

\begin{abstract}
An approach to calculation of reciprocal space maps of x-ray diffraction from partly relaxed multilayered epitaxial structures is reported. The theory takes into account the additional harmonics of wave field caused by the difference in the lateral projections of reciprocal vectors in the sample layers. The reciprocal space maps shown can be simulated on the basis of the dynamical diffraction theory and matrix method for boundary conditions, which are applicable to arbitrary experimental geometry. The developed theory explains the experimental results from several typical epitaxial structures in partly relaxed state.
\end{abstract}

DOI: 10.1103/PhysRevB.80.235315

PACS number(s): 61.05.cc, 61.05.cp, 68.65.Ac

\section{INTRODUCTION}

Crystalline epitaxial multilayers and superlattices play an essential role in modern nanoscale and semiconductor technologies. ${ }^{1}$ High-resolution x-ray diffraction (HRXRD) is an indispensable tool for investigation of the properties and quality control of these objects, such as layer thicknesses, relaxation degree, phase composition of the solid solutions, lattice deformations, etc. ${ }^{2}$ For the precise analysis of the HRXRD data, the two-wave approximation of the dynamical diffraction theory (DDT) is used to calculate the wave fields within the layers of the sample. ${ }^{3}$ The wave amplitudes are determined using either recurrent matrix equations ${ }^{4}$ or approximate Takagi-Taupin equations. ${ }^{5,6}$ In both cases, the boundary conditions are used consequently upon the continuity of wave fields at the interfaces of multilayers. ${ }^{3}$

The use of the conventional boundary conditions in DDT for the wave fields in the multilayered structures with different crystallographic lattice parameters is not justified in the case when the sample is no longer pseudomorphic, i.e., partly or fully relaxed. For example, let us consider the boundary (interface) between two crystalline layers in the diffraction plane, which is defined by the wave vector $\vec{k}$ of the incident wave and surface-normal vector $\vec{N}$, which is parallel to the axis $z$. The axis $x$ lies in the plane $z=0$. In the coplanar case, which is considered in this paper, the wave vectors $\vec{k}_{S}^{\prime}$ and $\vec{k}_{L}^{\prime}$ of the diffracted waves in the substrate $(S)$ and layer $(L)$ are also located in the diffraction plane. They correspond to the reflections from the crystallographic planes defined by the reciprocal space vectors $\vec{h}_{S}$ and $\vec{h}_{L}$ (Fig. 1). In more general cases, the lattice constants $a_{L}, c_{L} ; a_{S}, c_{S}$ are different for various layers and substrate (here we consider for the sake of simplicity the tetragonal unit cell). To characterize this difference, the dimensionless lateral $\xi_{\|}$and normal $\xi_{\perp}$ mismatches are usually introduced,

$$
\xi_{\|}^{R}=\frac{a_{L}^{R}-a_{S}}{a_{S}} ; \quad \xi_{\perp}^{R}=\frac{c_{L}^{R}-c_{S}}{c_{S}} .
$$

Here the index $R$ is related to the relaxed layer, which possesses the same lattice constants as a bulk crystal. The real values of the parameters $a_{L}, c_{L}$ within the multilayered structures depend however on the growth conditions and material properties, and are not equal to $a_{L}^{R}, c_{L}^{R}$. The crystallographic cell deformation of the layers is conditioned by the elastic properties of the material and interaction with the substrate, and is described by the degree of relaxation $R$ and mismatches,

$$
R=\frac{a_{L}-a_{S}}{a_{L}^{R}-a_{S}} ; \quad \xi_{\|}=\frac{a_{L}-a_{S}}{a_{S}} ; \quad \xi_{\perp}=\frac{c_{L}-c_{S}}{c_{S}} .
$$

The relaxation degree varies from $R=1$ (relaxed layer, $\xi_{\|}$ $\left.=\xi_{\|}^{R}\right)$ to $R=0$, i.e., $a_{L}=a_{S} ; \xi_{\|}=0$, which corresponds to pseudomorphic structure. The deformation of the unit cell is assumed to occur without cell volume changes, and thus the values $\xi_{\|}$and $\xi_{\perp}$ are interdependent through the Poisson ratio $\nu$. In the case of the tetragonal unit cell, this dependence is expressed as ${ }^{1}$

$$
\frac{c_{L}-c_{L}^{R}}{c_{L}^{R}}=-\frac{2 \nu}{1-\nu} \frac{a_{L}-a_{L}^{R}}{a_{L}^{R}} .
$$

The epitaxial films expose the complex microscopic structure owing to dislocations at their interfaces formed during the growth process. ${ }^{7}$ Within the kinematical theory of x-ray diffraction, the diffraction spectra from the structures possessing the dislocations have been calculated in Ref. 7 and recently extended in Refs. 8 and 9. The kinematical theory, however, has a limited applicability in the case of thick layers, involved in the diffraction process. At the same time, a simple approach based on the DDT and a single phenomenological parameter $R$ is proved to be effective, too. ${ }^{10,11}$ This approach however is only valid for the $\mathrm{x}$-ray diffraction from the pseudomorphic multilayered structures because of the equality of the reciprocal vector projections onto interfacial planes, $h_{x}^{0}=h_{S x}=h_{L x}=\cdots$ (Fig. 1). This fact makes it possible to separate the variables in the Maxwell equations and to reduce the problem to the sequence of one-dimensional differential equations (for example, Refs. 4-6).

The diffracted wave-vector components parallel to the surface are equal for all layers and are localized in a narrow angular interval $\Delta k_{x}^{\prime} / k_{x}^{\prime} \sim 1 / k_{0} L_{\|} \sim 10^{-7}$ close to the value $k_{x}^{\prime}=k_{x}+h_{x}^{0}$. Thus, the longitudinal component of the diffraction transfer vector $\vec{q}=\vec{k}^{\prime}-\vec{k}$ is localized in the vicinity of a single value for varying $\vec{k}$ (Fig. 1), 


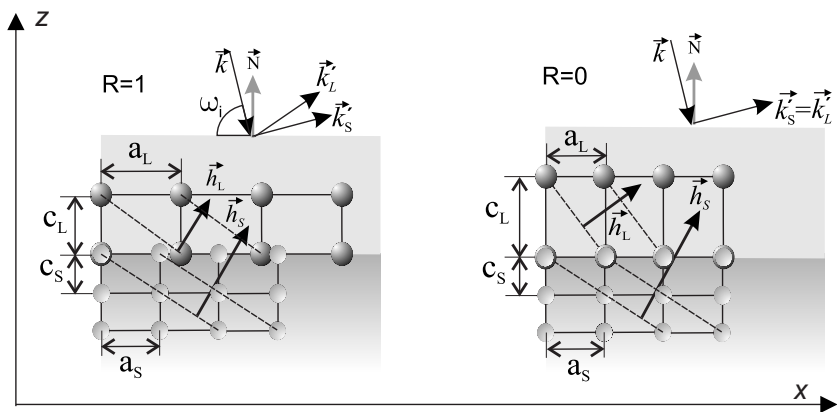

FIG. 1. X-ray diffraction from relaxed and pseudomorphic structures.

$$
q_{x}=k_{x}^{\prime}-k_{x}=h_{x}^{0} .
$$

In the case of the diffraction from the partly relaxed layer $(0<R<1)$, the values $a_{L} \neq a_{S} ; \xi_{\|} \neq 0$, i.e., $h_{S x} \neq h_{L x}$. Thus, the lateral components of the wave vectors are not equal $k_{x S}^{\prime} \neq k_{x L}^{\prime}$ and the waves reflected from the substrate and the layer propagate in different directions. Therefore, the additional harmonics $\exp \left[i l\left(h_{S x}-h_{L x}\right) x\right], l=0, \pm 1, \pm 2, \ldots$ (Fig. 2) have to be taken into account to provide a continuity of the wave fields at the interfaces. A determination procedure for the amplitudes of the harmonics has been proposed in Ref. 12 on the basis of DDT for infinite system of coupled algebraic equations and for the case of grazing-incidence geometry. The solution, however, is found in the approximation $\xi_{\perp}=0$, which is not satisfied for most structures owing to Eq. (3). An alternative approach has been considered in Ref. 13 based on the Takagi-Taupin equations, which fail in the case of grazing-incidence and extremely asymmetric diffraction.

In the present work, a method based on the DDT is developed for high-resolution $\mathrm{x}$-ray diffraction from partly relaxed multilayered structures. The approximate analytical solution for the wave fields which is satisfactory for the evaluation of HRXRD data is found for the entire range of the values of the lateral mismatch. This method allows the realization of an effective numerical algorithm for simulation of HRXRD reciprocal space maps (RSM) from the fully or partly relaxed epitaxial samples. The algorithm is also valid for the cases of grazing-incidence (exit) and extremely asymmetric diffraction (not considered in this work). In Sec. II, the algorithm for calculation of the amplitudes of the wave harmonics is developed for arbitrary variation in the lateral mismatches and the recurrent matrix formalism is extended to the case of the lateral lattice mismatches. In Sec. III, several numerical examples demonstrating the validity of the proposed technique are presented, and the method is applied to evaluation of the experimental data from typical semiconductor structures.

\section{X-RAY DIFFRACTION FROM THE MULTILAYERED PARTLY RELAXED SAMPLE}

Let us consider Bragg diffraction from the layer on the substrate with the lattice constants $a_{L}, c_{L}$ and $a_{S}, c_{S}$, respectively (Fig. 1). The propagation of $\mathrm{x}$ rays through the media can be considered in DDT (Ref. 3) separately for orthogonal

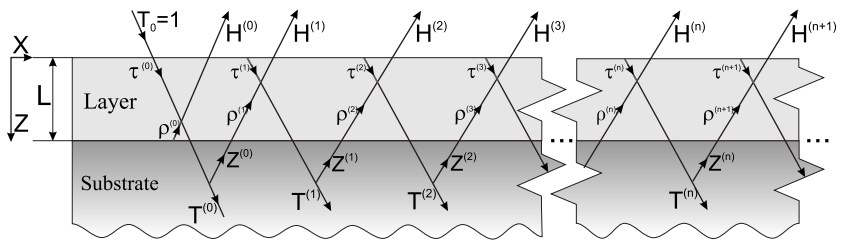

FIG. 2. The emergence of the additional harmonics in x-ray diffraction from the layer/substrate sample.

$\sigma$ and $\pi$ polarizations. For each polarization, the incident wave in vacuum is described by the function $T_{0} e^{i \overrightarrow{k_{0}} \vec{r}}$ with the scalar amplitude $T_{0}$ and the wave vector $\vec{k}_{0}$. The observed diffracted wave with the wave vector $\vec{k}^{\prime}$ is determined by the amplitude $U$. We assume here that $\vec{k}_{0}$ is close to the Bragg condition for both layer (reciprocal-lattice vector $\vec{h}_{L}$ ) and substrate (vector $\vec{h}_{S}$ ),

$$
2\left(\vec{k}_{0} \vec{h}_{L}\right)+h_{L}^{2} \approx 2\left(\vec{k}_{0} \vec{h}_{S}\right)+h_{S}^{2} \leq k_{0}^{2}\left|\chi_{0}^{L}\right| .
$$

Within the framework of the two-wave DDT, the incident wave excites inside the layer and substrate the transmitted waves with amplitudes $\tau_{1,2}^{(0)}$ and $T_{1,2}^{(0)}$, respectively, and the diffracted waves with amplitudes $\rho_{1,2}^{(0)}$ and $Z_{1,2}^{(0)}$. The wave field in crystalline layer is then expressed as ${ }^{3}$

$$
D_{L}^{(0)}(\vec{r})=\sum_{i=1,2}\left(\tau_{i}^{(0)}+\rho_{i}^{(0)} e^{\left.i \overrightarrow{h_{L}}\right)}\right) e^{i \overrightarrow{k_{i} \vec{r}}}
$$

where the wave vectors $\vec{k}_{i}, i=1,2$ and relations between amplitudes $\tau_{i}^{(0)}$ and $\rho_{i}^{(0)}$ are determined from the Maxwell equations taking into account the only three Fourier components of x-ray polarizability $\chi_{0}^{L}, \chi_{ \pm h}^{L}$. In the further equations, the case of the coplanar geometry is considered for the sake of simplicity, when the vectors $\vec{k}_{0}, \vec{N}, \vec{h}$ are located in the same plane. In this case, the vectors $\vec{k}_{0}, \vec{k}_{i}, \vec{h}_{S, L}$ have two components only and the projections of the wave vectors onto the sample surface are preserved (neglecting edge effects),

$$
k_{0 x}=k_{i x} \equiv k_{x}=k_{0} \cos \omega_{i},
$$

where $\omega_{i}$ is an incidence angle (Fig. 1). In the media, only the normal components of the wave vectors $k_{i z}$ are changed, which can be expressed through the dimensionless parameters $u_{i}$ (Ref. 4) as

$$
k_{i z} \equiv k_{0} u_{i}
$$

to be determined from the dispersion equation

$$
\left[u_{i}^{(0)^{2}}-\gamma_{0}^{2}-\chi_{0}^{L}\right]\left[\left(u_{i}^{(0)}+\psi^{L}\right)^{2}-\left(\gamma_{h}^{L}\right)^{2}-\chi_{0}^{L}\right]=C \chi_{\vec{h}}^{L} \chi_{-\vec{h}}^{L},
$$

with polarization factor $C=1$ for $\sigma$ polarization and $C$ $=\cos \left(2 \theta_{B}\right)$ for $\pi$ polarization. For the compactness reason, the case of $\sigma$ polarization is considered below. Additionally, the following parameters are introduced: ${ }^{14}$

$$
\gamma_{0}=\sin \omega_{i} ; \quad \psi^{L}=\frac{h_{z}^{L}}{k_{0}},
$$




$$
\begin{gathered}
\left(\gamma_{h}^{L}\right)^{2}=1-\frac{\left(k_{x}+h_{x}^{L}\right)^{2}}{k_{0}^{2}}=\gamma_{0}^{2}-2 \psi_{x}^{L} \sqrt{1-\gamma_{0}^{2}}-\left(\psi_{x}^{L}\right)^{2}, \\
\psi_{x}^{L}=\frac{h_{x}^{L}}{k_{0}}= \pm \sqrt{4 \sin ^{2} \theta_{B}^{L}-\left(\psi^{L}\right)^{2}},
\end{gathered}
$$

where $\theta_{B}^{L}$ is the Bragg angle for the layer. The essential difference of the Eq. (9) from the dispersion equation for pseudomorphic structure ${ }^{4}$ is the dependence of the parameter $\gamma_{h}^{L}$ on the layer physical properties. The amplitudes of the transmitted and diffracted waves are connected as

$$
\rho_{i}^{(0)}=v_{i}^{(0)} \tau_{i}^{(0)} ; \quad v_{i}^{(0)}=\frac{\chi_{\vec{h}}^{L}}{\left(u_{i}^{(0)}+\psi^{L}\right)^{2}-\left(\gamma_{h}^{L}\right)^{2}-\chi_{0}^{L}} .
$$

The dispersion Eq. (9) of the fourth order delivers four possible solutions for the wave vector in crystal but in twowave approximation the only two solutions are meaningful, for which $k_{i z} \approx k_{0 z}$. The two other roots with $k_{i z} \approx-k_{0 z}$ correspond to the specularly reflected waves with amplitudes, which are approximately $\left|\chi_{0}\right| \sim 10^{-5}$ times smaller than principal waves, if the grazing-incidence or grazing-exit diffraction cases ${ }^{4}$ are excluded.

In the similar way, the wave fields inside the substrate are calculated. For example, the wave with amplitude $\tau_{1,2}^{(0)}$, transmitted through the crystalline layer, excites inside the substrate the wave field consisting of the transmitted $T^{(0)}$ and diffracted $Z^{(0)}$ waves,:

$$
D_{S}^{(0)}(\vec{r})=\left(T^{(0)}+Z^{(0)} e^{\left.i \overrightarrow{h_{S}}\left(\vec{r}-\overrightarrow{r_{S}}\right)+i \phi_{n}\right)} e^{i \vec{k}_{i} \vec{r}}\right.
$$

where $\phi_{n}=\vec{h}_{L} \vec{r}_{S}{ }^{4}$ As regards two-wave DDT, these waves are expressed similarly to Eqs. (9) and (11),

$$
\begin{gathered}
Z^{(0)}=v^{(0)} T^{(0)} ; \quad v^{(0)}=\frac{\chi_{\vec{h}}^{S}}{\left(u^{(0)}+\psi^{S}\right)^{2}-\left(\gamma_{h}^{S}\right)^{2}-\chi_{0}^{L},} \\
{\left[u^{(0)^{2}}-\gamma_{0}^{2}-\chi_{0}^{S}\right]\left[\left(u^{(0)}+\psi^{S}\right)^{2}-\left(\gamma_{h}^{S}\right)^{2}-\chi_{0}^{S}\right]=\chi_{\vec{h}}^{S} \chi_{-\vec{h}}^{S} .}
\end{gathered}
$$

For the waves in the substrate, the additional boundary condition is applied, which implies the absence of the energy flux toward the substrate top border (there is no wave source with such a wave-vector direction). Therefore, one of the four solutions of Eq. (14) has to be chosen, for which $\mathfrak{R} u^{(0)}>0 ; \Im u^{(0)}>0 .{ }^{4}$ The amplitudes of the harmonics of the wave fields excited in the considered layer-substrate sample are displayed as an iterative sequence in Fig. 2. The diffracted wave $Z^{(0)}$ exiting the substrate excites in the layer the wave $\rho^{(1)}$ with wave vector equal to $\vec{k}^{S}+\vec{h}^{S} \approx \vec{k}_{0}+\vec{h}^{S}$. This wave vector satisfies the Bragg condition for crystallographic planes with reciprocal space vector $\left(-\vec{h}^{L}\right)$, which leads to the diffraction of the wave $\rho^{(1)}$ and yield of the amplitude $\tau^{(1)}$ corresponding to the wave vector $\vec{k}^{(1)} \approx \vec{k}_{0}+\vec{h}^{S}-\vec{h}^{L}$. All these waves satisfy the DDT equations analogous to Eqs. (6)-(9), with substitution of $k_{x}$ by $k_{x}+\left(h_{x}^{S}-h_{x}^{L}\right)$. In turn, the wave $\tau^{(1)}$ causes in substrate the wave fields $T^{(1)}, Z^{(1)}$ with the wave vectors equal $\vec{k}_{0}+\vec{h}^{S}-\vec{h}^{L}$ and $\vec{k}_{0}+2 \vec{h}^{S}-\vec{h}^{L}$, respectively. The wave $Z^{(1)}$ creates in the layer the waves $\tau^{(2)}, \rho^{(2)}$, with the wave vectors $\vec{k}^{(2)} \approx \vec{k}_{0}+2\left(\vec{h}^{S}-\vec{h}^{L}\right), \vec{k}^{(2)}+\vec{h}^{L}$, and so on (Fig. 2).

Thus, a set of wave harmonics is created where the DDT equations for amplitudes and wave vector of the $m$ th harmonic are obtained from Eqs. (6)-(9), (13), and (14) by substitution of $k_{x}$ by $k_{x}+m\left(h_{x}^{S}-h_{x}^{L}\right)$. In the general case, the diffracted wave $\rho^{(0)}$ may produce the wave fields $T^{(-m)}, Z^{(-m)}, \tau^{(-m)}, \rho^{(-m)}, m>0$, i.e., negative harmonics. They contribute essentially, however, only in the case of the grazing scattering geometry, when the specularly reflected waved have to be taken into account. An equation system for the amplitudes of the scattered $\mathrm{x}$-ray wave fields similar to the one described here has been obtained in Ref. 12 on the basis of the continuity of full wave field at the sample interfaces.

The recurrent relations between the amplitudes of the neighbor harmonics can be found using the boundary conditions with the preserving component of the wave vector parallel to the surface (Fig. 2),

$$
\begin{gathered}
\tau_{1}^{(n)}+\tau_{2}^{(n)}=0, \\
v_{1}^{(n)} \tau_{1}^{(n)}+v_{2}^{(n)} \tau_{2}^{(n)}=H^{(n)}, \\
\tau_{1}^{(n)} e^{i u_{1}^{(n)} t}+\tau_{2}^{(n)} e^{i u_{2}^{(n)} t}=T^{(n)} e^{i u^{(n)} t}, \\
v^{(n)} T^{(n)} e^{i u^{(n)} t}=v_{1}^{(n+1)} \tau_{1}^{(n+1)} e^{i u_{1}^{(n+1)} t}+v_{2}^{(n+1)} \tau_{2}^{(n+1)} e^{i u_{2}^{(n+1)} t},
\end{gathered}
$$

where dimensionless value $t=k_{0} L, L$ is the layer thickness, $H^{(n)}$ is the amplitude of the wave in vacuum, which is excited by the diffracted wave of $n$th harmonics. In Eq. (15) the expression $\chi_{\vec{h}}^{S}(\vec{r})=\chi_{\vec{h}}^{S} e^{i \vec{h}_{S}\left(\vec{r}-\overrightarrow{r_{S}}\right)+i \vec{h}_{L} \vec{r}_{S}}$ (Ref. 4) has been taken into account. Using Eq. (15) and the equations for $(n+1)$ harmonics, the expression for $\kappa^{(n)} \equiv H^{(n+1)} / H^{(n)}$ is found,

$$
\kappa^{(n)}=v^{(n)} \frac{v_{1}^{(n+1)}-v_{2}^{(n+1)}}{v_{1}^{(n)}-v_{2}^{(n)}} \frac{e^{i u_{1}^{(n)} t}-e^{i u_{2}^{(n)} t}}{v_{1}^{(n+1)} e^{i u_{1}^{(n+1)} t}-v_{2}^{(n+1)} e^{i u_{2}^{(n+1)} t}} .
$$

Taking into account the boundary conditions for zeroth harmonics $\rho_{1}^{(0)} e^{i u_{1}^{(0)} t}+\rho_{2}^{(0)} e^{i u_{2}^{(0)} t}=0, \tau_{1}^{(0)}+\tau_{2}^{(0)}=1$ (the amplitude $T_{0}$ of the wave in vacuum equals 1 ) the system of Eq. (15) is closed and leads to

$$
\begin{aligned}
& H^{(0)}=\frac{v_{1}^{(0)} v_{2}^{(0)}\left(e^{i u_{1}^{(0)} t}-e^{i u_{2}^{(0)} t}\right)}{v_{1}^{(0)} e^{i u_{1}^{(0)} t}-v_{2}^{(0)} e^{i u_{2}^{(0)} t}}, \\
& H^{(1)}=v^{(0)} \frac{v_{1}^{(1)}-v_{2}^{(1)}}{v_{1}^{(1)} e^{i u_{1}^{(1)} t}-v_{2}^{(1)} e^{i u_{2}^{(1)} t}} \frac{\left(v_{1}^{(0)}-v_{2}^{(0)}\right) e^{i\left(u_{1}^{(0)}+u_{2}^{(0)}\right) t}}{v_{1}^{(0)} e^{i u_{1}^{(0)} t}-v_{2}^{(0)} e^{i u_{2}^{(0)} t}}, \\
& H^{(n)}=H^{(1)} \prod_{i=1}^{n-1} \kappa^{(i)} .
\end{aligned}
$$

The expressions (16) and (17) define comprehensively the amplitudes of all harmonics. Below, the limiting cases are considered: $\xi_{\|} \approx 1$ and $\xi_{\|} \rightarrow 0$. In the former case, the diffrac- 

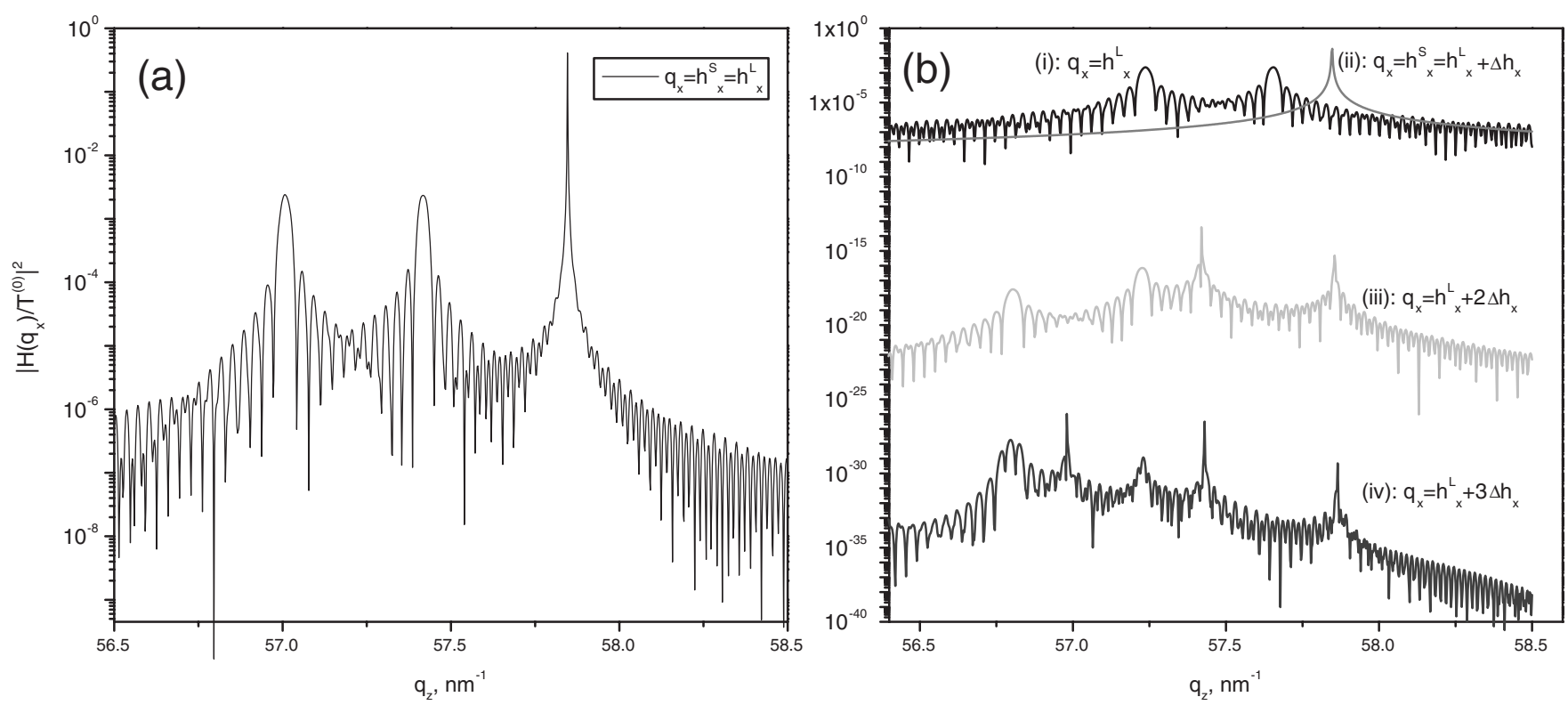

FIG. 3. Simulation of the $x$-ray diffraction intensity for the sample structure as described in the Table II (a) completely pseudomorphic multilayer $R=0$ and (b) with partly relaxed layers as designated in the Table II. The curves (i) and (ii) are the $q_{z}$ scans at different $q_{x}$ : $q_{x}$ $=h_{x}^{L}=36.4 \mathrm{~nm}^{-1}$ and $q_{x}=h_{x}^{S}=36.6 \mathrm{~nm}^{-1}$, respectively. The curves (iii) and (iv) demonstrate the contributions of the second and the third harmonics in the diffracted intensity.

tion on the layer and substrate can be considered independently, then $H^{(0)}$ and $H^{(1)}$ are the amplitudes of the wave diffracted on the layer and the substrate, respectively. Other harmonics may be neglected because of $\kappa \propto \chi_{\vec{h}}^{S} \chi_{\vec{h}}^{L}$ (in DDT, only the values $\geq\left|\chi_{\vec{h}}\right|$ are accounted for). In the latter case, all the harmonics leave the layer in the same direction, the values $\kappa^{(n)}$ are equal, and the amplitude of the diffracted wave $U$ in vacuum is

$$
\begin{aligned}
U & =H^{(0)}+H^{(1)}\left(1+\kappa+\kappa^{2}+\cdots\right) \\
& =H^{(0)}+\frac{H^{(1)}}{1-\kappa} \\
& =\frac{v_{1} v_{2}\left(e^{i u_{1} t}-e^{i u_{2} t}\right)+v\left(v_{1} e^{i u_{2} t}-v_{2} e^{i u_{1} t}\right)}{v_{1} e^{i u_{1} t}-v_{2} e^{i u_{2} t}-v\left(e^{i u_{1} t}-e^{i u_{2} t}\right)} .
\end{aligned}
$$

For a fully pseudomorphic structure (lateral mismatch equals zero), the conventional boundary conditions are applied without additional harmonics,

$$
\begin{gathered}
\tau_{1}+\tau_{2}=1, \quad v_{1} \tau_{1}+v_{2} \tau_{2}=U, \\
\tau_{1} e^{i u_{1} t}+\tau_{2} e^{i u_{2} t}=T e^{i u t}, \quad v_{1} \tau_{1} e^{i u_{1} t}+v_{2} \tau_{2} e^{i u_{2} t}=v T e^{i u t},
\end{gathered}
$$

that gives the $U$ presented in Eq. (18). Thus, the method developed above describes correctly both limiting cases of complete $(R=1)$ and zeroth $(R=0)$ relaxation of the layer on the substrate (Fig. 2).

To estimate the importance of the harmonics $H^{(2)}, H^{(3)}, \ldots$ in calculations, their contribution to the simulated HRXRD intensities has to be considered. At the scanning angles outside the Bragg-peak area where the deviation parameter $\alpha$ $\propto|\chi \vec{h}|$, the values $v^{(m)}, v_{1}^{(m)}, v_{2}^{(m)}$ are equal,

$$
v^{(m)} \propto \frac{\chi_{\vec{h}}^{S}}{\alpha_{S}^{(m)}}, \quad v_{1}^{(m)} \propto \frac{\chi_{\vec{h}}^{L}}{\alpha_{L}^{(m)}}, \quad v_{2}^{(m)} \propto \frac{\alpha_{L}^{(m)}}{\chi_{\vec{h}}^{L}}
$$

and

$$
H^{(2)} \propto H^{(1)} \frac{v^{(1)}}{v_{2}^{(1)}} \propto \frac{\chi_{\vec{h}}^{S}}{\alpha_{S}^{(0)}} \frac{\chi_{\vec{h}}^{S}}{\alpha_{S}^{(1)}} \frac{\chi_{\vec{h}}^{L}}{\alpha_{L}^{(1)}} .
$$

For the additional harmonics, the deviation from the Bragg condition depends not only on the lateral mismatch $\xi_{\|}$, tending to zero at $R=0$ but also on normal mismatch $\xi_{\perp}$, which is always larger than $\xi_{\perp}^{R}$ owing to Eq. (3). For the typical semiconductor solid solutions, $\xi_{\perp}$ varies within the limits $\xi_{\perp} \approx 10^{-3}-10^{-2}$, which are $10^{2}-10^{3}$ times larger than $\mathrm{X}$-ray susceptibility.

Assuming $\xi_{\|}=0$, the amplitude of the second harmonics in the vicinity of the layer peak is $H^{(2)} \propto\left(\chi_{\vec{h}}^{S} / \xi_{\perp}^{R}\right)^{2}$, as follows from Eq. (21). Thus $H^{(2)}$ is approximately three orders smaller than $H^{(1)}$. The harmonics of the higher order $H^{(3)}, \ldots$ are even smaller and rapidly decrease in the region far from the Bragg diffraction peaks. These estimates are confirmed by the numerical calculations for the contribution of the harmonics shown in Fig. 3(b) (lower curves). The simulations have been carried out for the reflection (135) at $\mathrm{Cu} K \alpha$ wavelength from the multilayered sample with the parameters described in the Table II, low intensity peaks from the thin Si layers are not taken into account. The Fig. 3(a) shows the diffraction from the same sample assuming the pseudomorphic nonrelaxed structure. The upper curves in the Fig. 3(b) demonstrate the diffracted intensity of the principal diffracted waves for layers and substrate. It should be noticed that both layers are partly relaxed and almost pseudomorphic relative to each other (the interference fringes appear). 
In the case of the partly relaxed layers [Fig. 3(b)], the intensities of the diffracted beams, determined by the amplitudes $H^{(0)}$ (layer) and $H^{(1)}$ (substrate), correspond to different values of $q_{x}$, and there is no interference between these scattering channels (the layer intensity displays the thickness oscillations). For the pseudomorphic case [Fig. 3(a)], the diffraction from the layers and substrate occurs at the same value of $q_{x}$, which causes a more complicated interference picture. As a result, the cross influence of the layer and substrate scattering exists in high-order harmonics at any relaxation degree but the amplitude of the harmonics is negligible and does not contribute to the HRXRD scans, in fact.

The analysis above confirms the main contribution to the detected HRXRD intensity by zeroth-order harmonics in each layer, which are for the considered sample: $T_{0}, H^{(0)}, H^{(1)}$ in vacuum; $\tau^{(0)}, \rho^{(0)}, \tau^{(1)}, \rho^{(1)}$ in layer; and $T^{(0)}, Z^{(0)}$ in substrate (Fig. 2). This fact allows to use a simplified algorithm instead of Eq. (15) for calculation of the amplitudes. The modified algorithm remains uniformly suitable for fully relaxed, partly relaxed, and pseudomorphic layers, which is an advantage in the case of multilayered structures.

Comparing the solutions of the simplified Eq. (19) with those found from Eq. (15), the following relations can be derived with the accuracy $O\left(\sim\left|\chi_{h}\right|^{2}\right)$ (pseudomorphic case):

$$
\begin{aligned}
\tau_{1}^{(0)} e^{i u_{1}^{(0)} t} & =T e^{i u^{(0)} t} ; \quad v_{2}^{(1)} \tau_{2}^{(1)} e^{i u_{2}^{(1)} t}=v T e^{i u^{(0)} t}, \\
H^{(0)} & =U-v T e^{-i u_{2}^{(1)} t} ; \quad H^{(1)}=v_{2}^{(1)} \tau_{2}^{(1)} .
\end{aligned}
$$

In the case of the partly relaxed layer, the amplitudes $H^{(0)}$ and $H^{(1)}$ determine the waves, which propagate in a vacuum in different directions and with the lateral projections of the wave vectors $k_{0 x}+h_{L x}$ and $k_{0 x}+h_{S x}$, respectively. If the equality $h_{S x}=h_{L x}$ holds, the total amplitude of these waves is equal to amplitude $U$ of the reflected wave for pseudomorphic structure. There is an evident physical interpretation of the formulas obtained above: the amplitude of the principal harmonics in the layer is obtained by the subtraction of the principal amplitude in the preceding layer from the amplitude in the layer in the pseudomorphic state, taking into account the wave phase owing to the refraction. The Eq. (22) expresses the wave amplitudes in partly relaxed layer through the solutions in an equivalent pseudomorphic layer. This fact simplifies the solution of the boundary problem (15), that is essentially for multilayered structures.

For investigation of the multilayered pseudomorphic structures, where $q_{x}$ is equal for all layers, the scanning of the intensity $I\left(q_{z}\right)$ over a single axis, e.g., $q_{z}$, is satisfactory. ${ }^{1}$ In the case of multilayered partly relaxed structures, however, the diffraction peaks from the layers possessing different mismatches are located at different positions of the scattering transfer vector components $q_{x}, q_{z}$. For such samples, the reciprocal space mapping over the coordinates $\left(q_{x}, q_{z}\right)$ or $(H, L)$ is used. The theoretical interpretation of these data usually utilizes either kinematical diffraction theory ${ }^{7}$ or approximate solutions of the Takagi-Taupin equations. ${ }^{10}$ However, as mentioned above, the kinematical theory is not applicable for the diffraction in thick layers and the TakagiTaupin equations fail for the diffraction in grazing-incidence or grazing-exit geometry. In opposite, the matrix method of $\mathrm{x}$-ray diffraction is applicable for arbitrary experimental geometry and number (thickness) of the layers in the sample ${ }^{4,15}$ but it demands the continuity condition for the lateral component of the wave vector at interfaces, which is satisfied for the pseudomorphic layers only. As demonstrated above, the additional harmonics of the diffraction field caused by different mismatches in layers contribute negligibly to the integrated $\mathrm{x}$-ray intensity. Basing on this fact, we develop an approach, which makes possible to simulate reciprocal space maps from the partly relaxed structures, keeping all the advantages of the matrix method for dynamical diffraction theory.

The algorithm proposed consists of the following steps. Assume the x-ray diffraction intensity has to be calculated from the structure composed of $N$ partly relaxed layers on the substrate. First, the simplified sample model is used with zeroth lateral mismatches $\xi_{\|}$. For this sample, the matrix method can be used for calculation of the wave-field amplitudes $D_{i, n}$ for $\pi$ and $\sigma$ polarizations for arbitrary number and thickness of the layers and arbitrary diffraction geometry. ${ }^{4,15}$ Here the index $i=1,2,3,4$ determines four roots of the dispersion Eq. (9) for $n$th layer; $n=0,1, \ldots, N+1$, where $n=0$ corresponds to vacuum and $n=N+1$ to substrate. Accordingly to Eq. (13), the amplitude of the diffracted wave in $n$th layer $U_{n}=\sum_{i=1}^{4} v_{i, n} D_{i, n}$ and the lateral component of the wave vector $k_{x}+h_{x}$ are equal for all the layers. In the partly relaxed sample, however, there exist the waves with the wave vectors $k_{x}+h_{x m}$ in each layer, besides the one with $k_{x}+h_{x n}$. These waves propagate in different directions and depend on the principal harmonics of the diffracted waves coming from the layers $m=(n+1), \ldots, N+1$ underneath (in substrate there exist only the principal wave). The amplitudes of all harmonics in the layers can be expressed through the solutions $D_{i, n}$ of the matrix equations for simplified pseudomorphic structure using the recurrent equations,

$$
\begin{gathered}
U_{n}^{(n)}=U_{n}-U_{n+1} e^{-i \varphi_{n}^{(n+1)}} ; U_{n}^{(n+1)} \\
=U_{n+1}^{(n+1)} e^{-i \varphi_{n}^{(n+1)}} ; \ldots U_{n}^{(N+1)} \\
=U_{N+1}^{(N+1)} e^{-i \varphi_{n}^{(N+1)}} ; \ldots \\
U_{N+1}^{(N+1)}=U_{N+1}, \\
U_{n}=\sum_{i=1}^{4} v_{i, n} D_{i, n}, \\
\varphi_{n}^{(m)}=k_{0} \sum_{l=n+1}^{m \geq n+1} u_{R}^{(l)}\left(k_{x m}\right) L_{l} .
\end{gathered}
$$

Here the subscript index in the values $U_{n}^{(m)}, n$ $=0,1, \ldots, N+1$ is a number of the layer and the superscript index $m=n+1, \ldots, N+1$ points [in opposite to as in the Eq. (15)] to the amplitude caused by the diffraction process in the underneath layer with the number $m ; L_{m}$ is the thickness of $m$ th layer; $\varphi_{n}^{(m)}$ is the phase owing to the refraction of the diffracted wave from $m$ th layer to $n$ layer. Parameters 
$u_{R}^{(l)}\left(k_{x m}\right)$ are the solutions of the dispersion Eq. (9) for $l$ th layer, corresponding to the reflected waves and wave vector $k_{x m}=k_{0 x}+h_{x m}$. Equation (23) is applicable to any ratio of the mismatches in the layers. In the general case of partly relaxed layers, the waves with amplitudes $U_{n}^{(m)}$ propagate at different angles, depending on the difference of the lateral projections of wave vector $k_{x n}-k_{x m}=h_{x n}-h_{x m}$. If all the mismatches are equal to zero (pseudomorphic case), all the reflected waves propagate in the same directions and

$$
\sum_{l=n}^{N+1} U_{n}^{(l)}=U_{n}
$$

thus the sum amplitude of the reflected waves coincides with the solution of the matrix equations for the pseudomorphic structure. ${ }^{4,15}$ The following result should be especially emphasized, despite the fact that the recurrent equations in Eq. (23) express the amplitudes in partly relaxed structure via the amplitudes in pseudomorphic one, the wave-field distribution is considerably different in these cases. Namely, in the former case there are $(N+1)$ reflected waves propagating with different projections of the wave vectors on the sample surface whereas in the latter case these projections for all the diffracted waves are equal.

Now we consider how the reciprocal-space-map intensity is calculated on the basis of the proposed method. Assume that a plane $\mathrm{x}$-ray wave with the wave vector $k_{x}, k_{z}$ $=\sqrt{k_{0}^{2}-k_{x}^{2}}$, incident on the sample, and the detector records the reflected radiation in a vacuum (Fig. 2) as a monochromatic wave with the wave vector $k_{x}^{\prime}, k_{z}^{\prime}$. Then the amplitude distribution is written in $k_{x}^{\prime}, k_{z}^{\prime}$ space as

$$
U\left(k_{x}^{\prime}, k_{z}^{\prime}\right)=\sum_{j=1}^{N+1} U_{0}^{(j)} \delta\left(k_{x}^{\prime}-k_{x}-h_{(j), x}\right) \delta\left(k_{z}^{\prime}+\sqrt{k_{0}^{2}-\left(k_{x}+h_{(j), x}\right)^{2}}\right) .
$$

Here the amplitudes $U_{0}^{(j)}$ are calculated using formula (23); the first $\delta$ function derives from transformation of the lateral component of the wave vector at each interface. The second $\delta$ function limits DDT to coherent elastic scattering without the change in the $\mathrm{x}$-ray wavelength.

The typical experimental scanning geometries for the measurement of the reciprocal space maps include $(2 \theta / \omega)$ (with relative or absolute $2 \theta$ and $\omega$ scales) and $(H, L)$ [or $\left(q_{x}, q_{z}\right)$ in other units] scanning modes. All the mentioned coordinates can be converted into each other, and therefore we consider here the simulation of the $(2 \theta / \omega)$ RSMs, where $\omega$ is an X-ray incidence angle on the sample surface and $2 \theta$ is the angle between incident beam and detector, and $q_{x}$ $=k_{0}[\cos (2 \theta-\omega)-\cos \omega] ; q_{z}=k_{0}[\sin (2 \theta-\omega)+\sin \omega]$. The incident beam usually has a certain divergence, described by the function $F_{B}\left(\omega-\omega_{B}\right)$, and the detector has a certain resolution, which is taken into account by the detector resolution function $F_{D}\left(2 \theta-2 \theta_{D}\right)$.

In the amplitudes of the wave fields derived above, each layer has been considered as an ideal crystal within the DDT concept. The real structure of the partly relaxed samples, however, is known to include the numerous dislocations. ${ }^{7}$ The diffuse $\mathrm{x}$-ray scattering from the defects results both in the diminished intensity of coherent (diffracted) waves and the broadening of the diffraction peaks. The microscopical approach to the analysis of the x-ray diffraction on the basis of the kinematical theory is developed in Ref. 7 and has recently been further extended in Refs. 8 and 9. The microscopical description of the diffuse x-ray scattering on the basis of the DDT matrix approach is a complicated task and is not considered in this work. The RSMs simulated below, however, include the incoherent contribution by using additional phenomenological parameters. The static DebyeWaller factors $e^{-W_{j}}$ take into account the reduction in the amplitude of the coherent wave in each layer caused by the diffuse x-ray scattering. ${ }^{7}$ The functions $F_{M}\left(\vec{q}-\vec{h}_{j}\right)$ describe the broadening of the diffraction peak owing to the diffuse scattering caused by the crystal defects. ${ }^{1}$

The observed $\mathrm{x}$-ray intensity of the scattered radiation in the reciprocal space point corresponding to the coordinates $\left(2 \theta_{D}, \omega_{B}\right)$ is obtained after the integration of the amplitude over the initial states and the convolution with the detector resolution function,

$$
\begin{aligned}
I\left(2 \theta_{D}, \omega_{B}\right)= & \int_{-\infty}^{\infty} F_{D}\left(2 \theta-2 \theta_{D}\right) \mid \sum_{j=1}^{N} F_{M}\left(\vec{q}_{D}-\vec{h}_{j}\right) \\
& \times\left. F_{B}\left[\omega_{j}(2 \theta)-\omega_{B}\right] U_{0}^{(j)} e^{-W_{j} / 2}\right|^{2} d(2 \theta),
\end{aligned}
$$

where $\omega_{j}(2 \theta)$ is found from $h_{(j), x}=q_{x}(\omega, 2 \theta)$ and is written as

$$
\begin{gathered}
k_{x}^{\prime}=k_{x}+h_{x} ; \\
\cos \left(2 \theta-\omega_{i}\right)=\cos \omega_{i}+\psi_{x j}, \\
\omega_{i}^{j}(2 \theta)=\frac{2 \theta}{2}+\arcsin \left\{\frac{\psi_{x j}}{2 \sin (2 \theta / 2)}\right\}, \\
q_{x D}=k_{0}\left[\cos \left(2 \theta_{D}-\omega_{B}\right)-\cos \omega_{B}\right] ; \quad q_{z D}=k_{0}\left[\sin \left(2 \theta_{D}-\omega\right)\right. \\
\left.+\sin \omega_{B}\right] .
\end{gathered}
$$

Thus the Eq. (26) being used for simulation of the diffracted x-ray intensity, utilizes the DDT for only the calculation of the amplitudes $U_{0}^{(j)}$, which corresponds to the pseudomorphic structure. In general case, these amplitudes differ from the ones delivered by the kinematical theory and determine the position and dynamical shape of the Bragg peaks without accounting the diffuse scattering caused by the defects in the crystal and the instrumental effects. For the analysis of the experimental data in the next section, the functions $F_{M}, F_{D}$, and $F_{B}$ are chosen as Gaussians with parameters obtained by the fitting of the measured reciprocal space maps. Because of the coherent processes are precisely taken into account within the framework of DDT, the fitting results deliver the averaged characteristics of the microstructural defects in the partly relaxed samples. This approach is certainly not entirely microscopic, however, it is effective enough for practical evaluation of the reciprocal space maps, as shown in the examples below. 


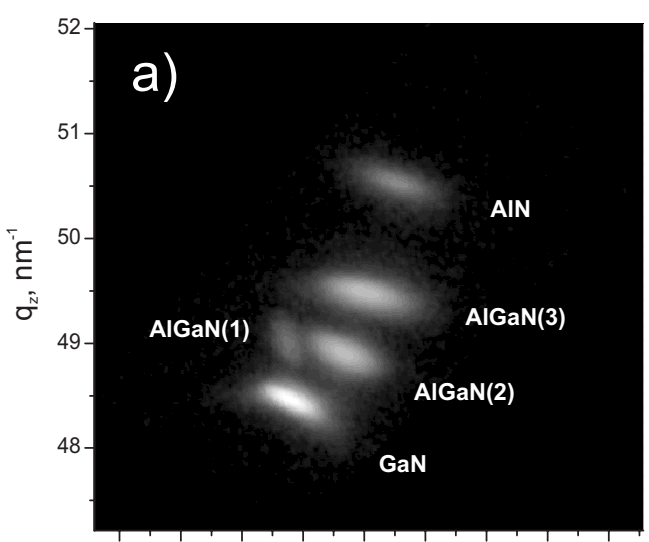

$\begin{array}{lllllllll}38.0 & 38.5 & 39.0 & 39.5 & 40.0 & 40.5 & 41.0 & 41.5 & 42.0\end{array}$ $\mathrm{q}_{\mathrm{x}}, \mathrm{nm}^{-1}$

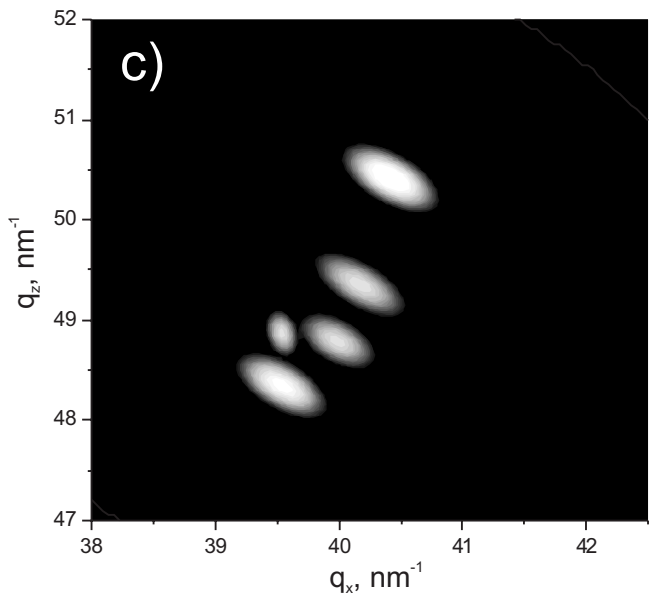

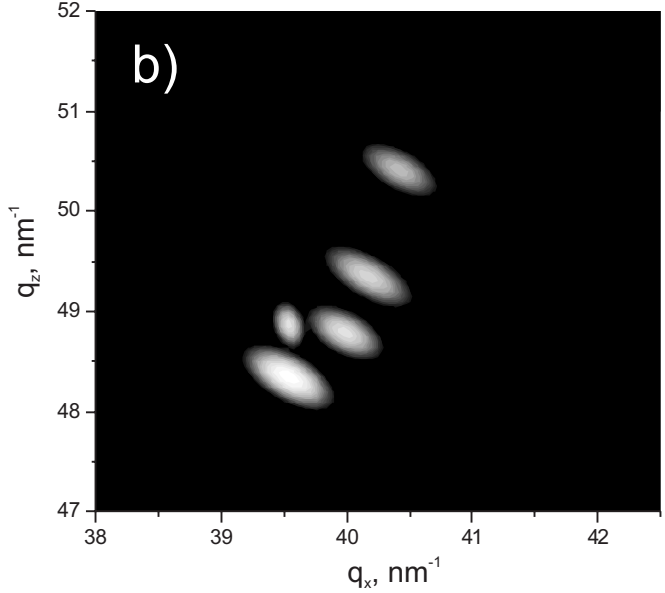

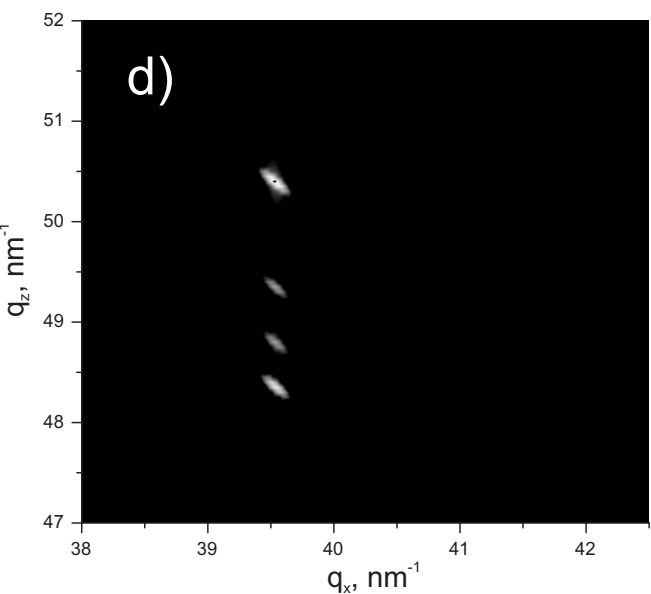

FIG. 4. $q_{x} q_{z}$ reciprocal space map for (114) Bragg reflection from AlGaN/GaN/AlGaN/AlN[Si] multilayer, described in the Table I: (a) experiment, (b) simulation with Debye-Waller factors $e^{-W_{j}}$, (c) simulation without incoherent scattering from dislocations, and (d) DDT simulation for the model sample with the pseudomorphic layers with the same thicknesses and concentrations.

\section{ANALYSIS OF EXPERIMENTAL DATA}

Figure 4(a) shows the experimental $q_{x} q_{z}$ RSM for (114) Bragg reflection of $\mathrm{Cu} K \alpha$ radiation from the sample composed of several $\mathrm{Al}_{x} \mathrm{Ga}_{1-x} \mathrm{~N}$ layers with different aluminum concentrations $x$ on the silicon $\mathrm{Si}(111)$ substrate, the substrate peak is not included in the measured area (sample by courtesy of Nitronex Corporation). Epitaxial structures such as this are being used as the starting material for the commercial production of $\mathrm{AlGaN} / \mathrm{GaN}$ high electron mobility transistors for various radio frequency applications. ${ }^{16,17}$

Figure 4(c) demonstrates the theoretically simulated map of the same (114) Bragg reflection on the basis of formula
(26), and Fig. 4(b) also includes a static Debye-Waller factor in simulations, which takes into account the decrease in the diffracted amplitude owing to the incoherent scattering from the dislocations. ${ }^{7}$

The resolution parameters $\Delta \omega_{B}, \Delta\left(2 \theta_{D}\right)$ have been estimated from the experimental setup whereas the layer parameters for the aluminum concentration $x$ and relaxation degree $R$ have been fitted with respect to the minimal difference between simulated and experimental peak positions. Table I shows the sample layer structure and the fitted sample parameters: layers thicknesses $t$, relaxation degree with respect to the previous layer $R$, the evaluated concentration $x$ of

TABLE I. Sample structure and layer parameters.

\begin{tabular}{lccccc}
\hline \hline Layer & Material & $\begin{array}{c}t \\
(\mathrm{~nm})\end{array}$ & $R$ & $x$ & $e^{-W}$ \\
\hline $\mathrm{AlGaN}(1)$ & $\mathrm{Al}_{0.20} \mathrm{Ga}_{0.80} \mathrm{~N}$ & 16 & 0.00 & 0.20 & 1 \\
$\mathrm{GaN}$ & $\mathrm{GaN}_{\mathrm{AlGaN}(2)}$ & 775 & 1.00 & & 1 \\
$\mathrm{AlGaN}(3)$ & $\mathrm{Al}_{0.31} \mathrm{Ga}_{0.69} \mathrm{~N}$ & 250 & 0.51 & 0.29 & 1 \\
$\mathrm{AlN}$ & $\mathrm{Al}_{0.58} \mathrm{Ga}_{0.42} \mathrm{~N}$ & 450 & 0.70 & 0.55 & 0.5 \\
& $\mathrm{AlN}^{\mathrm{Si} \text { substrate }}$ & 400 & 1.00 & & 0.02 \\
\hline \hline
\end{tabular}


TABLE II. Sample structure and layer parameters.

\begin{tabular}{lcrlc}
\hline \hline Layer & Material & $\begin{array}{c}t \\
(\mathrm{~nm})\end{array}$ & \multicolumn{1}{c}{$R$} & $x$ \\
\hline $\mathrm{Si}$ & $\mathrm{Si}$ & 23 & 0.0 & \\
$\mathrm{SiGe}(1)$ & $\mathrm{Si}_{0.89} \mathrm{Ge}_{0.11}$ & 200 & 0.08 & 0.10 \\
$\mathrm{Si}$ & $\mathrm{Si}$ & 6 & 0.0 & \\
$\mathrm{SiGe}(2)$ & $\mathrm{Si}_{0.79} \mathrm{Ge}_{0.21}$ & 180 & 0.65 & 0.20 \\
& $\mathrm{Si} \mathrm{substrate}$ & & & \\
\hline \hline
\end{tabular}

aluminum (the nominal value is displayed in the Material column), and static Debye-Waller factor.

The relaxation degree with respect to the previous layers $R$ is essentially different for the layers, which reflects the variation in the lateral mismatch $\xi_{\|} \neq 0$ within the multilayer. The working layer AlGaN(1) remains, however, strained because of the small layer thickness.

The AlN peak intensity experimentally observed in Fig. 4(a) is evidently smaller than that simulated on the basis of formula (26) in Fig. 4(c). This discrepancy can be explained by the mechanism of the relaxation in epitaxial structures with large lattice mismatches. According to Ref. 9, the lateral lattice periods of two crystalline layers are equal at their interface. This, however, is compensated by the appearance of the dislocation network close to the interface, which reforms the average lateral lattice parameter to the one, corresponding to the relaxed bulk state. In the vicinity of the dislocation nuclei the crystalline order is essentially broken but it is re-established toward the layer depth. In the case of the investigated sample, the $\mathrm{Si}(111)$ substrate and AlN layer in bulk state have the lateral lattice parameters $\sqrt{2} a_{\mathrm{Si}}$ $=0.768 \mathrm{~nm}$ and $a_{\mathrm{AlN}}=0.498 \mathrm{~nm}$. The dislocation networks appear to compensate for this large difference, distorting the crystalline order and resulting in the loss of the scattered coherent intensity. To take into account this effect in Eq. (26), additional static Debye-Waller factors $e^{-W_{j}}$ have to be introduced for diminution of the amplitude of the coherent diffraction owing to the incoherent scattering from the dislocations. ${ }^{7}$ Figure 4(b) shows the RSM calculated with the

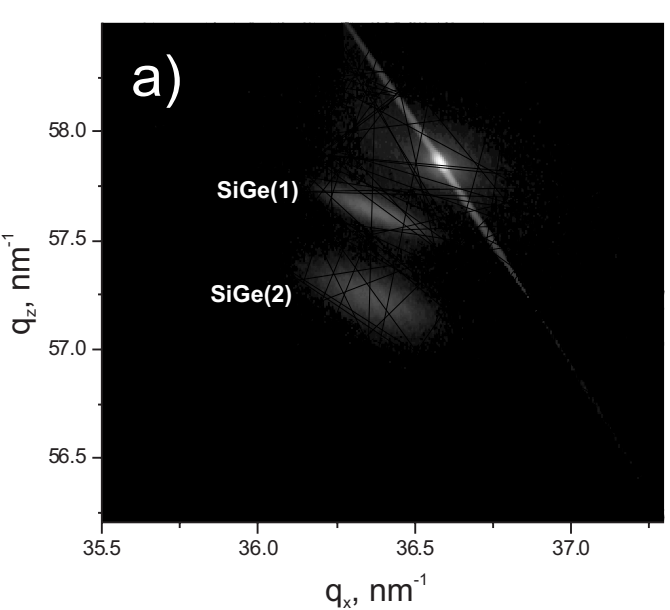

factors $e^{-W_{j}}$ for each layer listed in the Table I. The twisting and broadening of the peak intensities can be simulated by the functions $F_{M}(\vec{q}-\vec{h})$ and $F_{D}\left(2 \theta-2 \theta_{D}\right)$. Figure $4(\mathrm{~d})$ displays the simulated RSM on the basis of conventional DDT for the model structure without lateral mismatches and corrected for resolution effects, in this case the dislocations are absent and $e^{-W_{j}}=F_{M}(\vec{q}-\vec{h})=1$.

Figure 5 shows the (a) experimental and (b) simulated reciprocal space maps for (135) Bragg reflection collected from strained silicon structure described in Table II (sample by courtesy of Forschungszentrum Juelich, Juelich, Germany). The relative positions of the peaks on the simulated map are well situated in comparison with the measured intensities (very thin strained silicon layers are not observed due to weak scattering power). The relaxation degrees of both $\mathrm{Si}_{1-x} \mathrm{Ge}_{x}$ layers have been fitted, and the values are presented in Table II along with the evaluated concentrations of the germanium in the layers (the nominal concentrations are displayed in the material column). For simulated map, Fig. 5(b), the formula (26) has been used and the broadening of the peaks is mostly caused by the function $F_{M}(\vec{q}-\vec{h})$. The static Debye-Waller factor is close to unity for all the layers $e^{-W_{j}} \approx 1$, which is conditioned by the very similar values of the lattice parameters for both $\mathrm{Si}$ and $\mathrm{Ge}$.

\section{CONCLUSIONS}

The present work is a further ${ }^{14,15}$ extension of the matrix method for HRXRD. We developed X-ray dynamical diffraction theory from multilayered epitaxial structures, including partly relaxed samples. Being based on the matrix method, the technique also permits the simulations for arbitrary geometry of the experiment, including extremely asymmetric diffraction and grazing-incidence (exit) diffraction.

For calculation of the amplitudes of the wave field in the layers, the widely used model for the relaxation state of the epitaxial film is used, which is described by the relaxation degree $0 \leq R \leq 1$ or, equivalently, by normal and lateral lattice mismatches. In the framework of this model, the exact solution for the boundary problem comprising the continuity

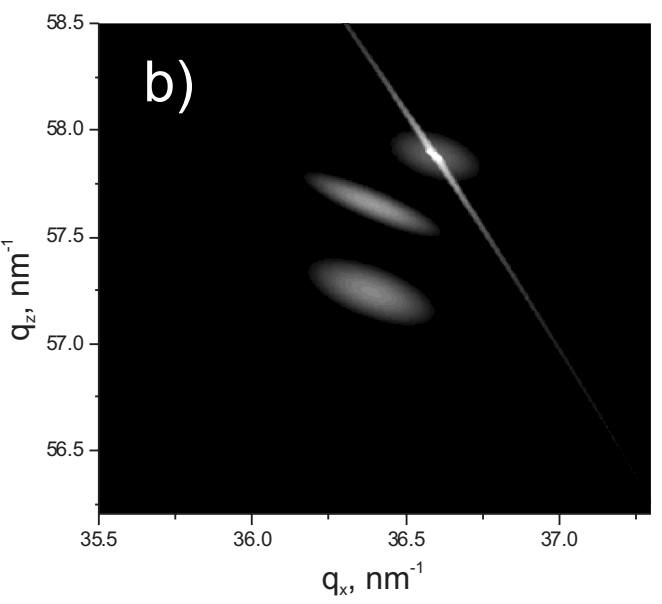

FIG. 5. $q_{x} q_{z}$ reciprocal space map for (135) Bragg reflection from $\mathrm{Si} / \mathrm{Si}_{0.89} \mathrm{Ge}_{0.11} / \mathrm{Si} / \mathrm{Si}_{0.79} \mathrm{Ge}_{0.21} /[\mathrm{Si}]$ multilayer, described in the Table II: (a) experiment; (b) simulation with the best-fitted relaxation degree of layers. 
of the wave fields at the interfaces is found. The principal harmonics in the layers are shown to be sufficient for the calculation of the intensity of the diffracted beams. The amplitudes of these harmonics in multilayered partly relaxed samples are expressed through the solutions of the recurrent equations for corresponding pseudomorphic layers, and thus the results are valid for the entire range of the relaxation parameter $R$.

The theory developed has been applied for the simulation of the high-resolution reciprocal space maps from the partly relaxed multilayered samples taking into account the extinction of the diffracted waves, the instrumental functions of the $\mathrm{x}$-ray source and the detector. The diffuse scattering from the dislocations presenting in the partly relaxed samples is simulated using a static Debye-Waller factor, which allows the relative intensities of the layer peaks to be fitted. The applicability of the method is demonstrated in the several experimental data and their simulations by the presented theory.

\section{ACKNOWLEDGMENTS}

The work is supported by the Ministry of Education of the Republic of Belarus (Projects "The fields and particles" and "Crystalline and molecular structures") and Bruker AXS GmbH (Germany). The authors are grateful to R. Loo (IMEC, Belgium), S. Mantl, and D. Buca (Institute of Bioand Nanosystems, Forschungszentrum Juelich GmbH, Germany) for providing strained silicon sample, and to J. Roberts (Nitronex Corporation, USA) for providing GaN-onSilicon sample.
${ }^{1}$ U. Pietsch, V. Holy, and T. Baumbach, X-Ray Scattering: From Thin Films to Lateral Nanostructures (Springer-Verlag, Heidelberg, 2004).

${ }^{2}$ Nondestructive Methods for Materials Characterization, edited by T. Matikas, N. Meyendorf, G. Baaklini, and R. Gilmore (Materials Research Society, Pittsburgh, 2000), Vol. 591.

${ }^{3}$ A. Authier, Dynamical Theory of X-Ray Diffraction (International Union of Crystallography, Oxford, 2003).

${ }^{4}$ S. A. Stepanov, E. A. Kondrashkina, R. Köhler, D. V. Novikov, G. Materlik, and S. M. Durbin, Phys. Rev. B 57, 4829 (1998).

${ }^{5}$ S. Takagi, Acta Crystallogr. 15, 1311 (1962).

${ }^{6}$ D. Taupin, Bull. Soc. Fr. Mineral. Cristallogr. 87, 469 (1964).

${ }^{7}$ M. A. Krivoglaz, X-Ray and Neutron Diffraction in Nonideal Crystals (Springer, Berlin, 1996), p. 101.

${ }^{8}$ V. M. Kaganer, R. Köhler, M. Schmidbauer, R. Opitz, and B. Jenichen, Phys. Rev. B 55, 1793 (1997).

${ }^{9}$ V. M. Kaganer, O. Brandt, A. Trampert, and K. H. Ploog, Phys. Rev. B 72, 045423 (2005).

${ }^{10}$ P. F. Fewster, X-Ray Scattering from Semiconductors, 2nd ed.
(Imperial College, London, 2002), p. 244.

${ }^{11}$ D. K. Bowen and B. K. Tanner, X-Ray Metrology in Semiconductor Manufacturing (CRC, Boca Raton/Taylor \& Francis, London, 2006), p. 60.

${ }^{12}$ A. P. Ulyanenkov, S. A. Stepanov, U. Pietsch, and R. Köhler, J. Phys. D 28, 2522 (1995).

${ }^{13}$ P. M. Fewster, J. Appl. Crystallogr. 25, 714 (1992).

${ }^{14}$ T. A. Alexeeva, A. I. Benediktovich, I. D. Feranchuk, T. Baumbach, and A. Ulyanenkov, Phys. Rev. B 77, 174114 (2008).

${ }^{15}$ I. D. Feranchuk, S. I. Feranchuk, A. A. Minkevich, and A. Ulyanenkov, Phys. Rev. B 68, 235307 (2003).

${ }^{16}$ Introduction to Nitride Semiconductor Blue Lasers and Light Emitting Diodes, edited by S. Nakamura and S. F. Chichibu (Taylor \& Francis, London, 2000).

${ }^{17}$ S. Singhal, A. W. Hanson, A. Chaudhari, P. Rajagopal, T. Li, J. W. Johnson, W. Nagy, R. Therrien, C. Park, A. P. Edwards, E. L. Piner, K. J. Linthicum, and I. C. Kizilyalli, International Conference on Compound Semiconductor Manufacturing Technology, Austin, TX, 2007, pp. 83-86. 